\title{
Special issue on: advances in multidimensional systems and signal processing
}

\author{
Krzysztof Galkowski · Nicholas P. Karampetakis
}

Published online: 26 July 2011

(C) The Author(s) 2011. This article is published with open access at Springerlink.com

The past five decades, in particular, have seen a continually growing interest in so-called two-dimensional (2D) or, more generally, multidimensional (nD) systems. This is clearly related to the wide variety of applications of both practical and/or theoretical interest. The key unique feature of an $\mathrm{nD}$ system is that the plant or process dynamics (input, output and state variables) depend on more than one indeterminate and hence information is propagated in several independent directions. Many physical processes have a clear nD structure. Also, the $\mathrm{nD}$ approach is frequently used as an analysis tool to assist, or in some cases enable, the solution of a wide variety of problems. A key point is that the applications areas for $\mathrm{nD}$ systems theory/engineering can be found within the general areas of Circuits, Control (with particular emphasis for Repetitive Processes and Iterative Learning Control) and Signal Processing (and many others). There are also still many unsolved problems in this area, solving of which requires new sophisticated mathematical methods and hence mathematical $\mathrm{nD}$ systems theory is still hot and developing extensively.

The objective of this special issue is to report some recent advances in multidimensional systems and signal processing, in both theoretical development and practical applications. The contributions in this special issue can be grouped into three thematic areas:

\section{Multidimensional systems}

The paper by E. Zerz provides a deeper insight into the study of behavior of finite generated and finite systems defined by linear differential equations with constant coefficients in a

Dedicated to the memory of Prof. Nirmal K. Bose.

K. Galkowski $(\varangle)$

Institute of Control and Computation Engineering, University of Zielona Góra, ul. Podgórna 50, 65-246 Zielona Góra, Poland

e-mail: K.Galkowski@issi.uz.zgora.pl

N. P. Karampetakis

Department of Mathematics, Aristotle University of Thessaloniki, 54124 Thessaloniki, Greece

e-mail: karampet@math.auth.gr 
Noetherian ring. J. Ball et al. identify how Livšic systems fit into the behavioral framework. This is achieved by extending the Oberst transfer matrix to the setting of autonomous behaviors and by making explicit identifications between the Oberst transfer matrix over the reduced ring and the Livšic Joint Characteristic function. D.S. Kaliuzhnyi-Verbovetskyi and $V$. Vinnikov survey the basic concepts of the theory of non-commutative rational functions, and their realization theory. Then, they develop a difference-differential calculus for noncommutative rational functions as a tool for the needs of system theory. L. Ntogramatzidis and $M$. Cantoni develop notions of conditioned invariant and detectability subspaces for 2D Fornasini-Marchesini models. This development leads to a Linear Matrix Inequality (LMI) based procedure for the synthesis of observers which asymptotically estimate the local state of a standard Fornasini-Marchesini model, in the sense that the error tends to zero as the reconstructed local state evolves away from unknown boundary conditions. N. Karampetakis and A. Evripidou present two interpolation algorithms for the computation of the inverse of a polynomial matrix. The first one is based on the Lagrange interpolation method, whereas the second one is using discrete Fourier transform techniques. D. Bors and St. Walczak provide sufficient conditions for the existence of an optimal control in the process of gas filtration that is described by a continuous $2 \mathrm{D}$ system.

\section{Repetitive processes}

T.P. Azevedo-Perdicoúlis and G. Jank present necessary and sufficient conditions for existence and uniqueness of an equilibrium of a N-player disturbed Nash game of linear quadratic type whose underlying structure is a repetitive process with smoothing. D. Idczak and $M$. Majewski present an existence theorem for a Lagrange optimal control problem connected with a two-directionally continuous variant of a linear autonomous repetitive process and convex integral cost functional. R. Rabenstein and P. Steffen show how the implicit discretization of partial differential equations can be cast into the framework of repetitive process. This very nice work brings the results from two different fields (control theory and numerical mathematics) together and presents it in a unified way. B. Cichy et al. apply iterative learning control (ILC) to systems that arise from Crank-Nicholson discretization of bi-variate partial differential equations describing spatio-temporal systems or processes.

\section{Multidimensional signal processing}

F. Belzen and $S$. Weiland initially propose a generalization of the Proper Orthogonal Decomposition (POD) method. Then by combining the techniques of tensor decomposition and POD they propose a model reduction framework for systems that have both space and time as independent variables. E. Skublska-Rafajlowicz and E. Rafajlowicz propose a new method of generating multidimensional deterministic sequences, which behave like uniformly distributed random vectors, but fill the space more evenly and therefore are well suited for $\mathrm{nD}$ sampling. F. Calliess et al. show how separability of general higher-order tensors can be leveraged to speed up multidimensional convolutions. J. Velten et al. present a new framework for realization of a general state-space description that is capable to consider non causal systems and shift operations with respect to more than one coordinate direction. This model offers better compliance with possible hardware realizations than existing models. A. Haselhoff and A. Kummert analyze a method of $\mathrm{nD}$ filtering based on prior signal 
integration. This method has the advantage that the computational complexity for filtering is independent of the filter kernel size.

This special issue is dedicated to the memory of Professor Nirmal K. Bose, Past Editorin-Chief of Multidimensional Systems and Signal Processing (MSSP), for his contribution to the general Multidimensional Systems area and also for supporting the idea of having this special issue. We would like to thank Profs. Z. Lin and A. Kummert, co-Editors of MSSP for their help during the preparation process, the authors who submitted papers for this special issue and the reviewers for their suggestions, comments and the time they spent for the evaluation of the papers. Finally, the kind help of Ms. Miriam Neethi from the Journal Editorial Office of Springer, is gratefully acknowledged.

Open Access This article is distributed under the terms of the Creative Commons Attribution Noncommercial License which permits any noncommercial use, distribution, and reproduction in any medium, provided the original author(s) and source are credited. 